\title{
ENERGY EFFICIENT DATA COMMUNICATION APPROACH IN WIRELESS SENSOR NETWORKS
}

\author{
${ }^{1}$ Jaswant Singh Raghuwanshi, ${ }^{2}$ Neelesh Gupta, ${ }^{3}$ Neetu Sharma \\ ${ }^{1}$ Scholar (M.Tech.), ECE Department,TIEIT, Bhopal \\ ${ }^{2}$ HOD \& A.P., ECE, Department,TIEIT, Bhopal \\ ${ }^{3}$ A.P., ECE, Department,TIEIT, Bhopal
}

\begin{abstract}
Wireless sensor network has a vast variety of applications. The adoption of energy efficient cluster-based configuration has many untapped desirable benefits for the WSNs. The limitation of energy in a sensor node creates challenges for routing in WSNs. The research work presents the organized and detailed description of energy conservation method for WSNs. In the proposed method reclustering and multihop data transmission processes are utilized for data reporting to base station by sensor node. The accurate use of energy in WSNs is the main challenge for exploiting the network to the full extent. The main aim of the proposed method is that by evenly distributing the energy all over the sensor nodes and by reducing the total energy dissipation, the lifetime of the network is enhanced, so that the node will remain alive for longer times inside the cluster. The result shows that the proposed clustering approach has higher stable region and network life time than Topology-Controlled Adaptive Clustering (TCAC) and Low-Energy Adaptive Clustering Hierarchy (LEACH) for WSNs.
\end{abstract}

\section{KEYWORDS:}

Stability period, Clustering, Energy efficiency, Network lifetime

\section{INTRODUCTION}

Energy efficient operation has been considered as one of the important challenge of wireless sensor networks (WSNs) for their practical applications. This energy conservation problem may become a constriction for many sensor networks consisting of battery-driven sensor devices where battery replacement is very costly or becomes almost impossible for many cases such as in the battlefield, building maintenance etc. In order to continue the network operability for long time a common design consideration must be focus on using some form of energy-efficient approaches[1]. One facet to consider for energy efficiency is the communication subsystem as it is the main energy consumer in WSNs [2]. It can consume 70\% of network energy. The energy consumption can be minimized efficiently through proper cluster head selection and message minimization in data reporting.

This paper, proposed energy efficient data communication approach in the context of clustering, where cluster heads are selected on the basis of remaining energy of sensor nodes and predefine probability. It increases the stability period of the network because cluster head needs more energy than normal nodes. Cluster head conserve energy to perform the operations like data reception, data aggregation and data transmission to base station, where as normal sensor node sense environmental conditions and send sense data to its respective cluster head. In the proposed 
International Journal of Advanced Smart Sensor Network Systems (IJASSN), Vol 4, No.3, July 2014

approach cluster head are selected in each round because rotation of cluster heads balance the energy in the network.

Cluster head selection is totally random and dynamic in this approach therefore it may happens more number of cluster heads are selected than the pre assumed value (predefine probability $x$ total sensor nodes). It consumed more energy in data transmission to base station. This paper proposed an approach in which cluster heads are regroup under one or more sensor nodes (second level cluster heads). This paper uses multihop transmission to transmit data from second level cluster head to cluster head and base station, vice versa.

The proposed approach is fully distributed, provides non-overlapping cluster heads in network. It attains minimum energy consumption for intra cluster communication with a significant reduction in the variation of the number of cluster heads selected per round compared with LEACH [3]. The simulation results show that the proposed approach has longer stable region and network life time than TCAC and LEACH.

The rest of the paper is organized as follows. In Section 2, this paper present related work. In Section 3, it Proposed approach for energy conservation in WSN is describes. In Section 4, it provides simulation results and performance analysis of Proposed approach. In Section 5, it concludes this paper.

\section{LITERATURE REVIEW}

In wireless sensor network energy conservative clustering approach is a key problem to increase the network lifetime. It is also difficult to create energy efficient clustering approach due to the completed energy configuration and network operation. In clustering, cluster heads require extra energy for receiving data from cluster member nodes and sending aggregated data to base station, which can be located far from the sensor network. Rotating the role of cluster heads among sensor nodes helps to distribute the loads evenly among all nodes in the clusters. Many energy efficient routing protocols are designed around clustered network structure[4][5]. The selection of cluster heads can be done based on many criteria such as node residual energy, node id, nodes' proximity to other nodes or randomness[3]. The cluster heads' selection is determined independently and probabilistically by each senor node. In the LEACH protocol, cluster heads are selected based on a random round robin [3]. Each node calculates its probability to become a cluster head and generate a random number between 0 and 1 . If the number is less than the calculated probability, the sensor node will become cluster head. The protocol forces every sensor node to become a cluster head regardless of the energy level of the sensor node.

In PEGASIS, sensor nodes will be organized in the network as a chain, only one sensor node of the chain aggregate all data and send it to the sink [6]. The problem in this protocol is that it based on the requirement of the global knowledge of the network topology. The HEED protocol is another distributed cluster based routing protocol in which the selection of cluster head dependent upon the residual energy of the sensor node and also selects these cluster heads stochastically [4]. In EECS which chooses the cluster heads with more residual energy through local radio communication [7]. In cluster formation phase, EECS considers the tradeoff of energy expenditure between sensor nodes to the cluster heads and the cluster heads to the base station. But, it increases the need of global knowledge about the distance between the cluster heads and the base station. In DEEC the cluster heads are selected on the basis of probabilistic threshold based on the ratio between remaining energy of sensor nodes and the average network energy [8]. DEEC does not require any global knowledge of energy at every election round. It can also well perform in multilevel heterogeneous sensor networks. In EECRP the Base Station selects the 
International Journal of Advanced Smart Sensor Network Systems (IJASSN), Vol 4, No.3, July 2014

Cluster Heads for sensing field[9]. The cluster head selection procedure is carried out in two stages. In the first stage, all the sensor node become candidate nodes for becoming $\mathrm{CH}$ are listed, based on the parameters like relative distance of the candidate node from the Base Station, residual energy level, possible number of neighboring sensor nodes the candidate node can have, and the number of times the candidate node has already become the Cluster Head. The EECRP protocol is centralized, which consume lot of energy in communication between sensor nodes and base station for parameter collection. A battery aware distributed clustering and routing protocol (BADCR) incorporates the state of the battery's remaining charge and health parameters in computing the charge utility metric at each cluster formation round[10]. BADCR shows that by optimizing the charge expenditure along with the nonlinear dynamics of the battery through the various degrees of freedom, significant improvement in energy efficiency is achieved. The Topology-controlled adaptive clustering scheme features capability of cluster heads to adjust their power level to achieve optimal degree and maintain this value throughout the network operation[11]. In the TCAC a topology control allows an optimal degree, which results in a better distributed sensors and well-balanced clustering system enhancing network lifetime [11]. In TCAC multihop communication does not used for data reporting to base station. It needs a distributed approach to consider parameter as many as possible.

\section{1. PROPOSED APPROACH}

This section, presents Proposed approach for wireless sensor networks. The following assumptions with respect to the Proposed approach are made.

\subsection{System model and assumptions}

The following assumptions are made for the sensor networks:

I.All sensor nodes and base station remain stationary. After deployment all the sensor nodes and sink remain stationary.

II. The sensor nodes are arbitrarily distributed over sensing field.

III.The sensor nodes are densely deployed in the sensing field. This dense deployed network achieves Quality of service.

IV.Sensor nodes recognize the relative position of the base station in the field.

All sensor nodes are homogeneous in terms of power, communication and processing capabilities.

V.Sensor nodes are able to measure the present energy level. The sensor nodes have no awareness of their location.

VI.A network operation model similar to [3][11] is adopted here, which consists of rounds. Each round consists of a complete cycle of cluster head selection, cluster formation and followed by data collection phase

\subsection{The Proposed Approach}

Proposed approaches accomplish in 3 phases setup phase I, setup phase II and steady condition phase. In setup phase I cluster head is selected on the basis of probabilistic threshold (based on remaining energy). At the setup phase II all the cluster heads are selected and formation of cluster is accomplished. In steady condition phase reclustering process starts where all the selected cluster heads reselect the second level cluster heads using threshold which is based on nodes remaining energy and distance to base station. After the completion of second phase second level 
International Journal of Advanced Smart Sensor Network Systems (IJASSN), Vol 4, No.3, July 2014

cluster heads receive aggregated data from nearest cluster heads (red lines) and again aggregate this data then send it to the base station (green lines) as shown in figure 1. This prolongs the stability period of network. It is very crucial for many applications where stability of network needed due to reliability.

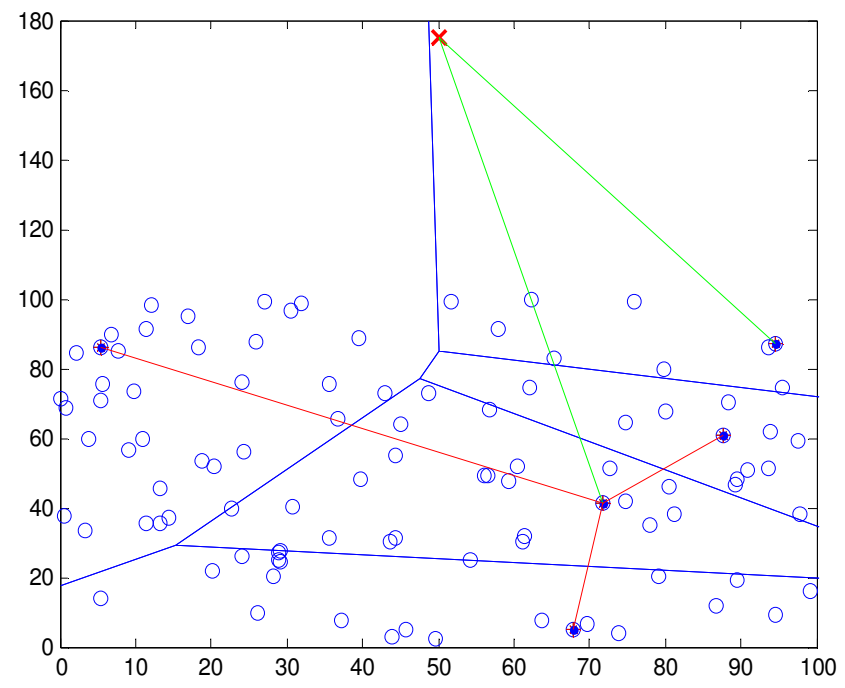

Fig 1: The clustering in Proposed approach

\subsubsection{Set up phase I}

The setup phase I includes cluster head election and cluster formation process. In the cluster head election process each sensor node chooses a random number between 0 and 1 separately. If this number is lower than the calculated threshold $T(i)$ for sensor node $i$, then the sensor node i become a cluster head.

The threshold $T(i)$ is given by

$$
T(i)=\left(\frac{\mathrm{P}_{\mathrm{c}}}{\left(1-\mathrm{P}_{\mathrm{c}} \times \bmod \left(\mathrm{r}, \operatorname{round}\left(1 / \mathrm{P}_{\mathrm{c}}\right)\right)\right)}\right) \times \frac{\mathrm{E}_{\mathrm{rem}}}{\mathrm{E}_{\max }}
$$

Where

$P_{c}$ is the optimal probability for initial cluster head ris the current round $E_{\text {rem }}$ is the remaning energy of sensor node $\mathrm{E}_{\text {max }}$ is the maximum or initial energy of sensor nodes

In the cluster formation process each cluster heads broadcast a cluster formation message within the sensing field. On reception of cluster formation message each non cluster head sensor node decide to join the cluster head, if it received the cluster formation messages more than one then sensor node join nearest cluster head. After a constant time interval cluster head received cluster join request messages from non cluster head sensor nodes. It creates a TDMA schedule for data transmission within the cluster and sends to its cluster members 


\subsubsection{Steady condition phase and Setup phase II}

The steady condition phase and setup phase II includes reselection of cluster heads and data aggregation process. In the reselection process second level cluster heads are selected on the basis of threshold and random number. The threshold $\mathrm{T}(\mathrm{s})$ for leaders shown below:

$$
\mathrm{T}(\mathrm{s})=\left(\frac{\mathrm{P}_{\mathrm{sc}}}{\left(1-\mathrm{P}_{\mathrm{sc}} \times \bmod \left(\mathrm{r}, \operatorname{round}\left(1 / \mathrm{P}_{\mathrm{sc}}\right)\right)\right)}\right) \times \frac{\mathrm{E}_{\mathrm{rem}}}{\mathrm{E}_{\max }} \times \frac{\mathrm{D}_{\max }}{\mathrm{D}}
$$

Where

$\mathrm{P}_{\mathrm{sc}}$ is the optimal probability for second level cluster heads

$\mathrm{D}_{\text {max }}$ is the maximum distance of sensing field

Dis the distance between sensor node and base station

After the second level cluster heads selection, cluster head are regroups under the newly selected second level cluster heads. Each second level cluster heads broadcast the regroup message within the sensing field. Cluster head join the nearest second level cluster head by sending confirm message. After a constant time each second level cluster head create a TDMA schedule for cluster head and send to its respective members cluster head. Each cluster head send aggregated data at the time slot allocated to it.

In the data aggregation phase each non cluster head sensor nodes send sense data to its respective cluster head in the allocated time period. The cluster head aggregates data received by its cluster members and send it to the respective second level cluster head in the allocated time period. If there is any other cluster head node on the way to second level cluster head then it send data using multihop. Cluster head send data to nearest cluster head which send data to second level cluster head. Each second level cluster head receive data from its member cluster heads and aggregated it then send this data to the base station using multihop communication. The clustering process of Proposed approach is shown above in figure 2. 
International Journal of Advanced Smart Sensor Network Systems (IJASSN), Vol 4, No.3, July 2014

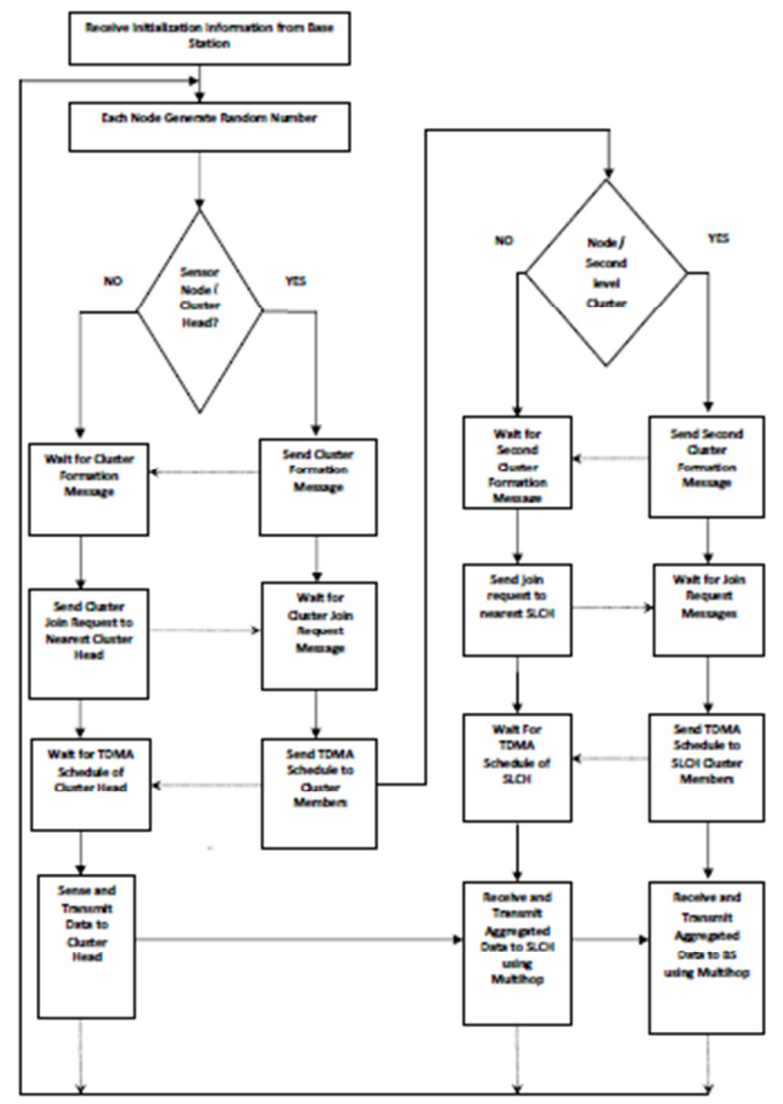

Fig 2: The flow chart of Proposed approach

\section{Simulation Result}

Implementation of proposed approach with clustered wireless sensor network in a field with dimension. The total number of sensors node $n=100$. The sensor nodes are randomly distributed over the sensing field. It means that vertical coordinate of each sensor nodes are arbitrarily selected between 0 and maximum value of dimension. The sink is outside the sensing field. The initial energy of a sensor node is set to $0.5 \mathrm{~J}$. The radio characteristic uses in our simulation are summarized in table 1.

\subsection{Stability period and Network lifetime}

The stability period and network lifetime are used as key indicators to estimate performance of the proposed approach. The stability period shows that the time interval from the start of the operation to the first node dies. Stability period of the Proposed approach is about the 1070 rounds as shown in figure 3 (a). The network lifetime is the time interval from the start of operation to the last node dies. Network lifetime of Proposed approach is greater than 2000 rounds as shown in figure 3 (a). The data transmissions from sensor nodes were simulated until all the sensor nodes died. 
International Journal of Advanced Smart Sensor Network Systems (IJASSN), Vol 4, No.3, July 2014 Table 1: Simulation Parameters

\begin{tabular}{|c|c|}
\hline Parameter & Value \\
\hline Network size & $\left(100 \times 100 \mathrm{~m}^{2}\right)$ \\
\hline Number of sensor node $(\mathrm{n})$ & 100 \\
\hline Base station position & $(50 \mathrm{~m}, 175 \mathrm{~m})$ \\
\hline Initial energy & $0.5 \mathrm{~J}$ \\
\hline$P_{\mathrm{c}}$ & 0.05 \\
\hline$P_{\mathrm{sc}}$ & 0.2 \\
\hline Transmitter Receiver electronics $\mathrm{E}_{\mathrm{elec}}$ & $50 \mathrm{nj} / \mathrm{bit}$ \\
\hline Data aggregation $\left(\mathrm{E}_{\mathrm{DA}}\right)$ & $5 \mathrm{nj} / \mathrm{bit} / \mathrm{packet}$ \\
\hline Reference distance $\left(\mathrm{d}_{0}\right)$ & $87 \mathrm{~m}$ \\
\hline Transmit amplifier $\epsilon_{\mathrm{fs}}$ & $10 \mathrm{pJ} / \mathrm{bit} / \mathrm{m}^{2}$ \\
\hline Transmit amplifier $\epsilon_{\mathrm{mp}}$ & $0.0013 \mathrm{pJ} / \mathrm{bit} / \mathrm{m}^{4}$ \\
\hline Message size $(\mathrm{l})$ & $4000 \mathrm{bits}$ \\
\hline
\end{tabular}

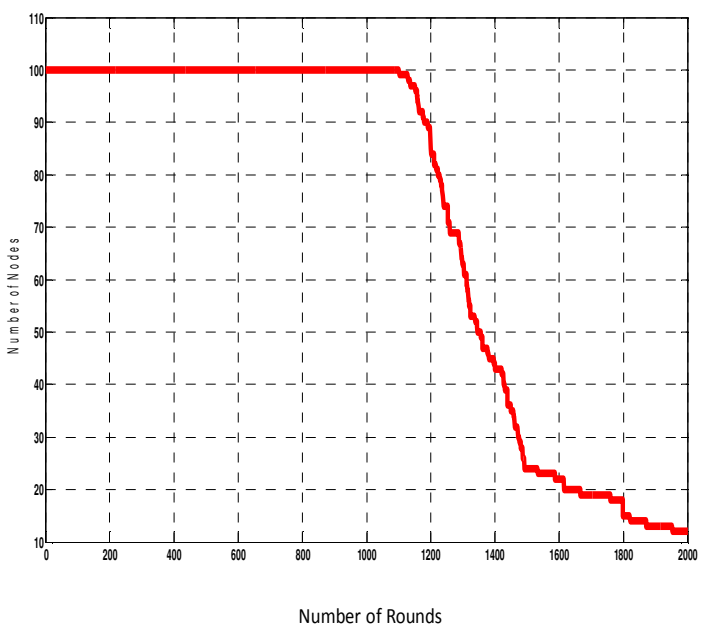

(a) 


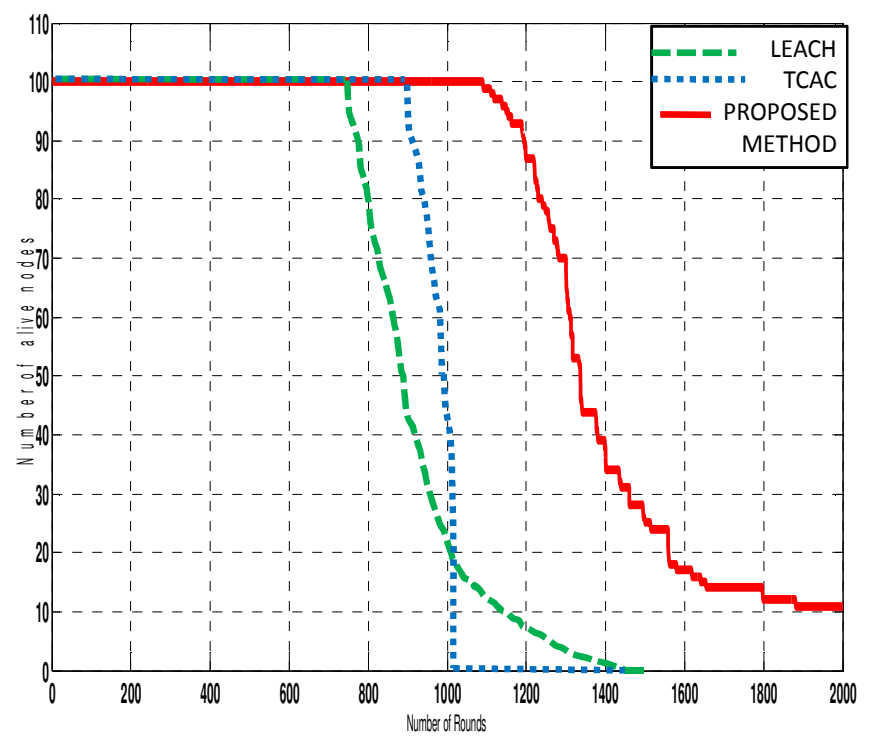

(b)

Fig 3: The performance of Proposed approach in term of stability period and network lifetime

Figure 3 (b) shows the comparative results of LEACH, TCAC and Proposed approach for network stability period and network lifetime [11]. The stable region of Proposed approach is about 1070 rounds which is larger than LEACH [3](760 rounds) and TCAC (870 rounds) stable region[11]. The network lifetime of LEACH is larger than TCAC protocol. In TCAC last node is die around 1050 round but in case of LEACH protocol until 1500 rounds 5 nodes are still alive. The network lifetime of Proposed approach is longer than LEACH protocol as shown in figure 3. The observation has been made that the Proposed approach is best for applications needed reliability because it has higher stability period as well as higher network lifetime.

\subsection{Number of Packet transmission from cluster head to Sink}

After the cluster election, each cluster head send out aggregated data to sink. Here the analysis is made on cluster formation and packet transmission. The figure 4 shows that the number of clusters formed in a round up to 47 clusters, but figure 5 shows that the number of packet transmitted to base station is lower than the clusters formed in field. 
International Journal of Advanced Smart Sensor Network Systems (IJASSN), Vol 4, No.3, July 2014

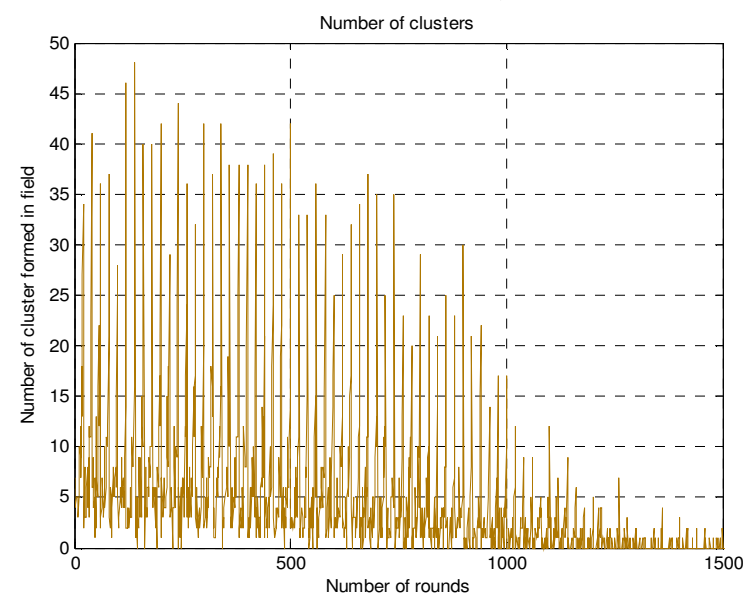

Fig 4: The formation of cluster in per round in Proposed approach

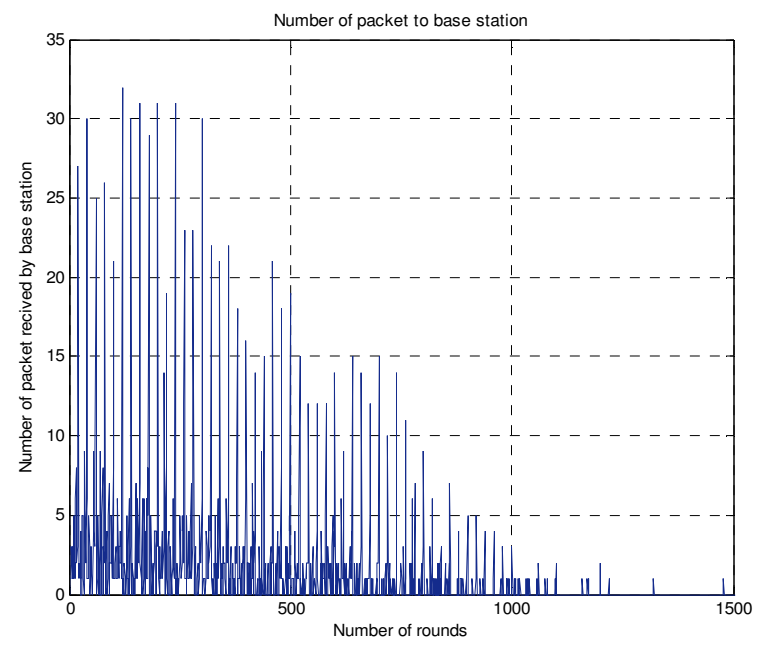

Fig 5: The packets received by sink in per round in Proposed approach.

\subsection{Probability for initial cluster head}

The probability for initial cluster head used to calculate the threshold for cluster head selection. It determines the number of cluster head in each round. We observe the performance of proposed approach by varying the value of $\mathrm{k}_{\text {initial }}\left(\mathrm{k}_{\text {initial }}=\right.$ predefine probability $\times$ total sensor nodes $)$. We observed that by increasing the value of $\mathrm{k}_{\text {initial }}$, numbers of cluster heads are increases therefore stability of network is also increases. Figure 6 shows that stability period of TCAC protocol increase from 880 rounds to 955 rounds[11].After some values TCAC stability period decreases because intercluster communication increase. In Proposed approach stability period increases from 1070 rounds to 1843 rounds. 
International Journal of Advanced Smart Sensor Network Systems (IJASSN), Vol 4, No.3, July 2014

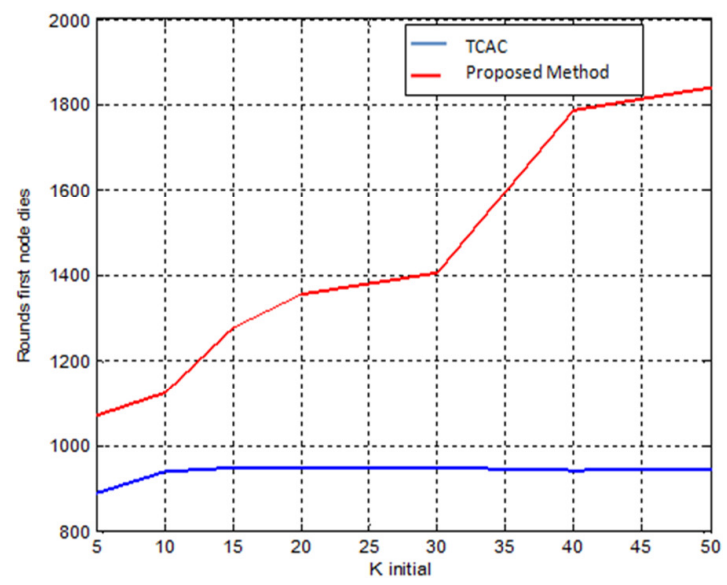

Fig 6: Stability period for different $\mathrm{K}_{\text {initial }}$

\section{CONCLUSiON}

In wireless sensor networks energy conservation is directly related to the network lifetime and also energy play an important role in the cluster head selection. A new threshold has been formulated for cluster head selection which is based on the remaining energy of the sensor node and the distance from base station. Proposed approach selects cluster head nearer to base station having maximum remaining energy than any other sensor nodes. This approach minimizing the inter cluster communication without effecting the data reliability. The main objective of this paper is to find ways to distribute the cluster head throughout the sensing field that will improve energy efficiency and help in reliable transmission of sensed data to the base station in wireless sensor networks. So it can say that proposed approach is best for the homogenous WSNs due to the higher efficiency of this routing protocol.

\section{FUTURE SCOPE}

WSNs is quite a burning issue in wireless communication means that much research is going on and many more issues are subjected to be investigated in this domain. Due to the time limitations, proposed approach work focus was only on the energy efficient clustering Approach. Though, there are many possible directions needed to be explored. The future directions for proposed approach vary from network structure to, application types to application demands. Different applications have different sensitivity factors. Different network designs have different constraints with respect to varying challenges.

$>\quad$ Different challenges need to be implemented on different protocols in real scenarios to identify protocols efficiencies.

$>\quad$ Clustering protocols need to be evaluated with specific performance metrics with respect to application demand in order to identify protocols suitability for different applications.

$>\quad$ Clustering protocols protection should be investigated with respect to various natures of attacks to which wireless communication is considered as an attractive target.

$>\quad$ QoS for applications in WSNs needs to explored and appropriate algorithms need to be devolved.

$>\quad$ The effect of the undesired mobility is studied in another set of experiments. 
International Journal of Advanced Smart Sensor Network Systems (IJASSN), Vol 4, No.3, July 2014

\section{REFERENCES}

[1] A. . Al-Karaki, J.N., Kamal, "Routing techniques in wireless sensor networks:a survey," IEEE Wirel. Commun., vol. 11, no. 6, pp. 6-28, 2004.

[2] C. Dargie, W., Poellabauer, 'Fundamental of wireless sensor networks, theory and practice. Wiley, 2010.

[3 ]W. B. Heinzelman, A. P. Chandrakasan, S. Member, and H. Balakrishnan, "An Application-Specific Protocol Architecture for Wireless Microsensor Networks,” vol. 1, no. 4, pp. 660-670, 2002.

[4] O. Younis and S. Fahmy, "HEED $\square$ : A Hybrid , Energy-Efficient, Distributed Clustering Approach for Ad-hoc Sensor Networks," vol. 0238294, pp. 1-36, 2004.

[5] A. A. Abbasi and M. Younis, "A survey on clustering algorithms for wireless sensor networks," Comput. Commun., vol. 30, no. 14-15, pp. 2826-2841, Oct. 2007.

[6] and C. S. R. Lindsey, Stephanie, "PEGASIS: Power-efficient gathering in sensor information systems," in Aerospace conference proceedings IEEE, 2002.

[7] M. Ye, C. Li, G. Chen, and J. Wu, "EECS $\square$ : An Energy Efficient Clustering Scheme in Wireless Sensor Networks," in In Perfomance, Computing, and Communications Conference IPCCC 2005. 24th IEEE International, 2005, pp. 535-540.

[8] L. Qing, Q. Zhu, and M. Wang, "Design of a distributed energy-efficient clustering algorithm for heterogeneous wireless sensor networks," vol. 29, pp. 2230-2237, 2006.

[9] S. P. Barfunga, "Energy Efficient Cluster Based Routing Protocol for Wireless Sensor Networks," no. July, pp. 3-5, 2012.

[10] J. Rao and A. O. Fapojuwo, "A Battery Aware Distributed Clustering and Routing Protocol for Wireless Sensor Networks,” pp. 1538-1543, 2012.

[11] P. Multimedia, "Topology-controlled adaptive clustering for uniformity and increased lifetime in wireless sensor networks," vol. 2, no. January 2011, pp. 318-327, 2012.

[12] Liao Ying, Huan Qi and Weiqun Li, "A Load-Balanced Clustering Algorithm with Distributed SelfOrganization for Wireless Sensor Networks", in Sensors Journal, IEEEVolume:13 , Issue: 5, pp.1498-1506, 2013.

[13] Ranjani, S. S., S. R., Krishnan, and C., Thangaraj, "Energy-efficient Cluster Based Data Aggregation for Wireless Sensor Networks." International Conference on, In Recent Advances in Computing and Software Systems (RACSS), vol., no., pp. 174-179, 25-27 April 2012, IEEE 2012.

[14] S.Prasanna and Srinivasa Rao "An Overview of Wireless Sensor Networks Applications and Security" International Journal of Soft Computing and Engineering (IJSCE) Volume-2, Issue-2, May 2012.

\section{AUTHORS:}

Jaswant Singh Raghuwanshi is a M.Tech Scholar from Truba institute of Engineering \& Information Technology, Bhopal .His Area of interest is Wireless Communication. He complete his BE in Electronics \& Communication (2007) from TCT - Thakral College of Technology,Bhopal.India

Neelesh Gupta is Pursuing Ph.D in Electronics and Communication from Rajiv Gandhi Technical University (RGTU), Bhopal (M.P.) India. He has a rich experience of teaching in various Technical institutions of reputed in MP, India. Recently he has submitted their PhD work. He is having more than 12 years of teaching Experience. Presently he is working as HoD, ECE in Truba Institute of Engineering and Information Technology (T.I.E.I.T.), Bhopal

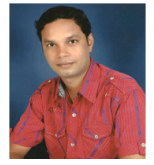

(M.P.) India. Total 15 students have completed their PG degree under his supervision. He has earned his BE in Electronics and Telecommunication in 2002 from LNCT, Bhopal and MTech degree in Microwave and Millimeter Wave in 2007 from MANIT, Bhopal. His area of research Interests are Wireless Communication, Microwave and Digital Signal Processing. He has presented a number of research papers in various National and International conferences in India and Abroad. He has Published number of Papers in International Journals. He is Life time member of IETE, New Delhi (M.P.). 
International Journal of Advanced Smart Sensor Network Systems (IJASSN), Vol 4, No.3, July 2014

Neetu Sharma is working in Truba Institute of Engineering \& Information Technology ,Bhopal as a Assistant Professor since July 2007 to till date. She has Awarded "Gold Medal" for being the topper of MTECH (EC) in the 7th convocation of Rajiv Gandhi Technical University, Madhya Pradesh. She has done her M. Tech in VLSI Design (May 2011) from SSSIST- Sri Satya Sai Institute of Science \& Technology College, Sehore

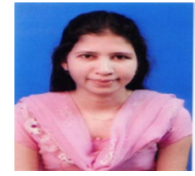
affiliated to RGPV - Rajiv Gandhi Technical University, Bhopal (M.P.) with 79.07\% aggregate. And BE in Electronics \& Communication (2007) from TCT - Thakral College of Technology, Bhopal (M.P.) affiliated to RGPV - Rajiv Gandhi Technical University, Bhopal (M.P.) with 78\% aggregate. She attends 8 workshops, 5 international conference \& 6 national conferences. Her 4 journal and 2 book published. 